\title{
INVESTIGATION OF DEVICE FOR GEOPHYSICAL EXPLORATION AT SHALLOW DEPTH
}

\author{
Obbozzhon Kuldashev \\ Fergana branch of Tashkent University of Information Technologies \\ 185 Mustaqillik str., Fergana, Uzbekistan, 150118 \\ kuldashov.abbos@mail.ru \\ Ibrohimzhon Tojiboev \\ Fergana branch of Tashkent University of Information Technologies \\ 185 Mustaqillik str., Fergana, Uzbekistan, 150118
}

\begin{abstract}
The article presents the results of the development of the echolocation device for geophysical exploration at shallow depth. The principle of operation, block diagram and time diagrams of the echolocation device for geophysical exploration at shallow depths are outlined. The urgency of developing an echolocation device for geophysical exploration at shallow depth is revealed in the conditions of a lack of mineral resources, the need to replenish them with the help of discovering new deposits.

It is analyzed that under the action of a shock wave, multi-frequency harmonic damped seismic waves are excited to the earth's surface, the frequency band of which is $1-200 \mathrm{~Hz}$.

When detonation generators are used to control the earth's crust, the duration of the impact pulse is on the order of one millisecond and the frequency range at 0.5 from the maximum amplitude of the echo signals is $20-400 \mathrm{~Hz}$, which allows using higher-frequency harmonic components of echo signals to determine and classify the object at depth up to $100 \mathrm{~m}$.

Echolocation device for geophysical exploration is intended for detection and primary classification of objects by their acoustic rigidity and can be used for geophysical exploration at shallow depths (up to $100.0 \mathrm{~m}$ ).

Keywords: geophysical exploration, echolocation device, block diagram, principle of operation, timing diagrams, detonation generator, echo signal.
\end{abstract}

\section{Introduction}

Today, one of the most urgent areas of geology and geophysics, are methods of studying the earth's crust.

In the conditions of a lack of mineral resources, the need to replenish them with the discovery of new deposits, new effective methods for studying the earth's crust acquire special significance and are very relevant [1-3].

\section{Materials and methods of research}

The aim of research is to study the condition and properties of rocks, to monitor the environment during the forecast of undesirable geodynamic phenomena (landslides, collapses, karsts, etc.). The task of research is to develop a method and device for monitoring the earth's crust.

For terrestrial shallow explorations, a variety of geophysical methods are used: seismic, electro- and magnetic exploration, nuclear methods and thermometry [4]. Features of shallow research in comparison with other types of exploration geophysics are related to the specifics of the tasks and the conditions of work. Shallow geophysics is widely used to control various operations performed in the construction and maintenance of structures [5].

A feature of shallow geophysics is the need to use modifications and types of seismic investigations associated with the use of acoustic (frequency above $0.5-1.0 \mathrm{kHz}$ ) and ultrasonic (more than $20 \mathrm{kHz}$ ) frequency ranges of elastic vibrations. Regardless of the working conditions, in terrestrial shallow geophysics tend, as a rule, to register waves of different types: longitudinal, transverse, exchange, surface, so shallow seismic exploration is essentially a multiwave. In works $[1,6]$ the technique of seismic exploration, based on the use of quasi-sinusoidal vibrations is given (vibroseis, acoustic sounding). 
Acoustic and ultrasonic studies are used for profiling in mine workings, translucence and logging. In addition, ultrasonic measurements are carried out on rock samples. In the study of rock massifs, acoustic and ultrasonic logging, profiling along the walls of mine workings, and ultrasonic observations in boreholes - shallow wells (up to 4-6 m) specially drilled in the walls of mine workings are most often used. The diameter of the holes is $50-60 \mathrm{~mm}$. For profiling along the holes and translucence through them, multichannel borehole probes with a pitch of $10 \mathrm{~cm}$ are used. Piezoelectric transducers are used as the source and receiver of oscillations [5].

In the study of sand clay rocks, ultrasonic methods are used for measurements in pits, excavations, ditches, etc., as well as for logging. In all cases, where possible, measurements are taken with the joint use of translucence and profiling. With the help of X-ray transmission, it is possible to obtain the most reliable data on the velocity of longitudinal waves. Profiling is used to determine the velocity of Rayleigh waves and the subsequent calculation of the velocity of transverse waves. The greatest difficulties are associated with providing a reliable sensor-rock contact, since a strong clamp can change the rock properties [9].

Electrical exploration is widely used for studying ground, artesian, fissured-karstic and mineral waters, and also for studying the conditions for watering mineral deposits [9].

In work to control the earth's crust at shallow depth, an acoustic method of control is applied. To assess the nature of the object, a phase shift is measured between two neighboring harmonic components of the echo signals, which differ in frequency. The choice of a pair of harmonic components of echo signals is made by their more intense amplitudes, which in seismic exploration with the use of powerful explosive sources correspond to the frequency range of $20-80 \mathrm{~Hz}$.

\section{Research results}

A device is proposed that includes a synchronizer connected to the indicator start input, a radiation source, a radiation receiver, to which the input of the first and second filter is connected. The output of the second filter is connected through the first amplifier-limiter and frequency doubler with the first input of the phase detector, the second input of which through the second amplifier-limiter is connected to the output of the second filter and the indicator. The input is connected to the output of the phase detector and additional threshold devices are added. The input of the threshold device is connected to the output of the radiation receiver. A sequentially connected trigger with separate startup, a time meter and an additional indicator start the connection input with the synchronizer output.

The first input of the trigger with separate startup is connected to the output of the threshold device, and the second one is connected to the output of the second amplifier-limiter, and according to the indication of the indicator, the classification of the object is determined. According to the indications of an additional indicator, the depth of the object from the surface of the earth is determined. In this case, the radiation source is made in the form of a detonation generator. The first and second solenoid valves controlling the inputs are connected to the output of the single oscillator. The input of the first solenoid valve is connected to the outlet of the oxygen source, and the input of the second solenoid valve is connected to the outlet of a source of gaseous hydrocarbon fuel. The outputs of the solenoid valves are connected to the corresponding inlet of the mixer of the hydrocarbon-air mixture of the detonation generator. The ignition device of the detonation generator is connected through an initiation system and a short pulse generator with a single oscillator output. The input of the single oscillator is connected to the output of the synchronizer. A cooler is installed inside the mixer of the hydrocarbon air mixture of the detonation generator, and the combustion chambers of the detonation generator are provided with cooling radiators, the length of the detonation chamber determined from the conditions

$$
\mathrm{L}_{\mathrm{dc}} \leq \frac{\mathrm{V}_{\mathrm{d}}}{\mathrm{V}_{\mathrm{e}}} 2 \mathrm{~h}_{\text {min }},
$$

where $\mathrm{h}_{\text {min }}$ - the minimum depth of the sought object; $\mathrm{V}_{\mathrm{d}}$ and $\mathrm{V}_{\mathrm{e}}$ - the velocity of propagation of the detonation wave and seismic waves in the earth, respectively. 
The block diagram of the proposed device is shown in Fig. 1.

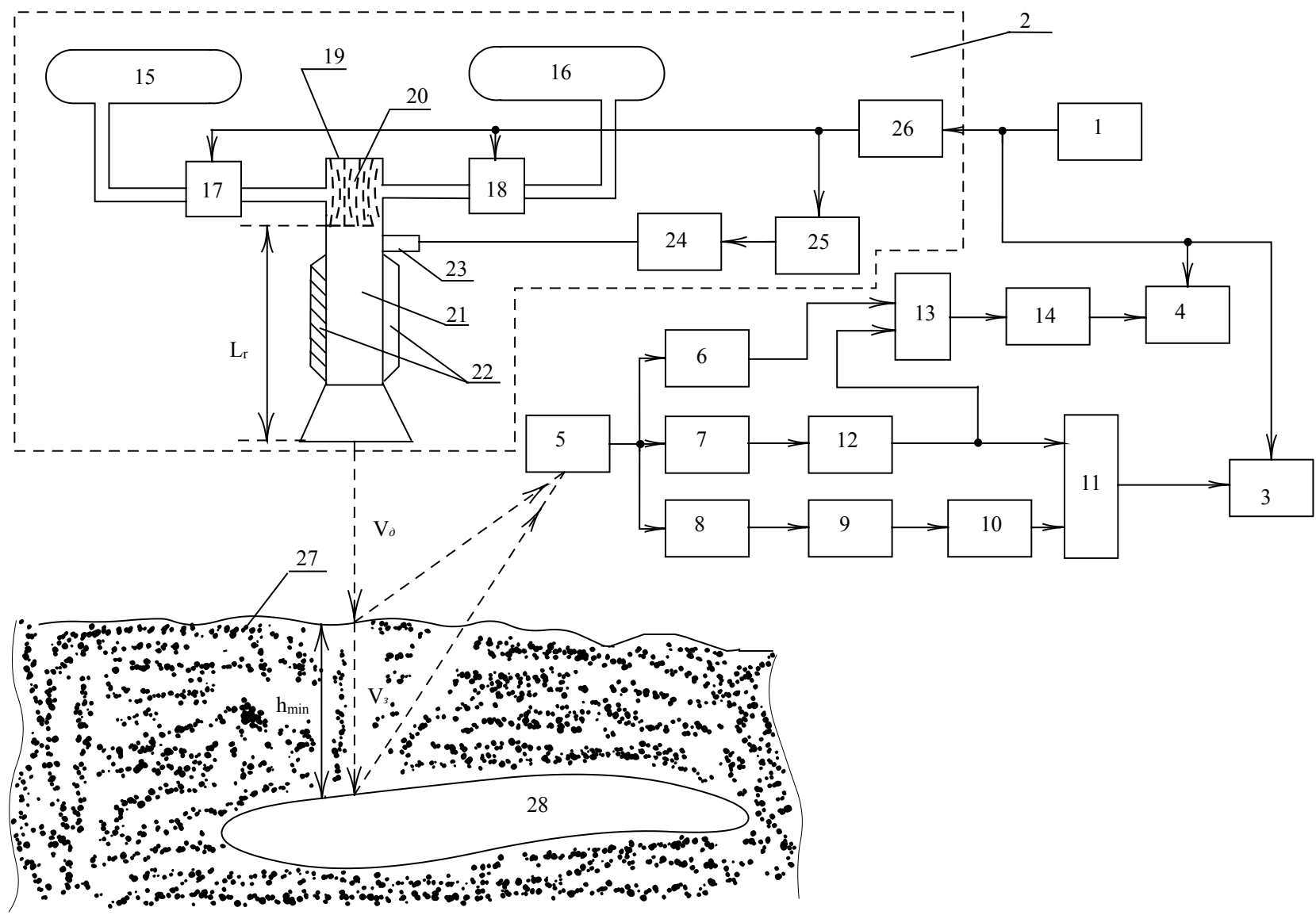

Fig. 1. Block diagram of the device: 1 - synchronizer, 2 - radiation source, 3 - first indicator, 4 - second indicator, 5 - radiation receiver 6 - threshold device, 7 - first filter, 8 - second filter, 9 - amplifier-limiter, 10 - frequency doubler, 11 - phase detector, 12 - amplifier, 13 - trigger,

14 - time interval meter, 15 - oxygen source, 16 - source of gaseous hydrocarbon,

17, 18 - solenoid valves, 19 - hydrocarbon air mixture mixer, 20 - cooler, 21 - detonation generator, 22 - radiator, 23 - device, 24 - initiation system 25 - short pulse generator, 26 - single oscillator, 27 - ground surface, 28 - search object

The device works as follows. The synchronizer 1 generates a sequence of short rectangular pulses with a repetition period $\mathrm{T}>\frac{2 \mathrm{~h}_{\max }}{\mathrm{c}}\left(\mathrm{h}_{\max }-\right.$ the maximum depth of the observation, $\mathrm{c}-\mathrm{ve}-$ locity of propagation of acoustic waves in the medium) which acts on the triggering inputs of the acoustic radiation source 2, the first indicator 3, the second indicator 4 and the time meter 14 .

As a result, the first 3 , the second 4 indicators and the time interval meter 14 are resetting information and preparing to receive the next acoustic information, and a rectangular pulse is generated at the output of the single oscillator 26, which is supplied to the trigger inputs of the short pulse generator 25, the solenoid valve of the gaseous hydrocarbon fuel 18 and solenoid valve for compressed air 17.

In this case, the open time of the solenoids 17 and 18 corresponds to the duration of the rectangular pulses generated by the single oscillator 26 , which are sufficient to completely fill the chamber of the detonation generator 21 by hydrocarbon air mixture.

At the end of the output pulse of the single oscillator 26, at the output of the short-pulse generator 25, a short rectangular pulse is formed (Fig. 2, $\boldsymbol{b}$ - time $\mathrm{t}_{1}$ ) which acts on the input of the initiation system 24 . 


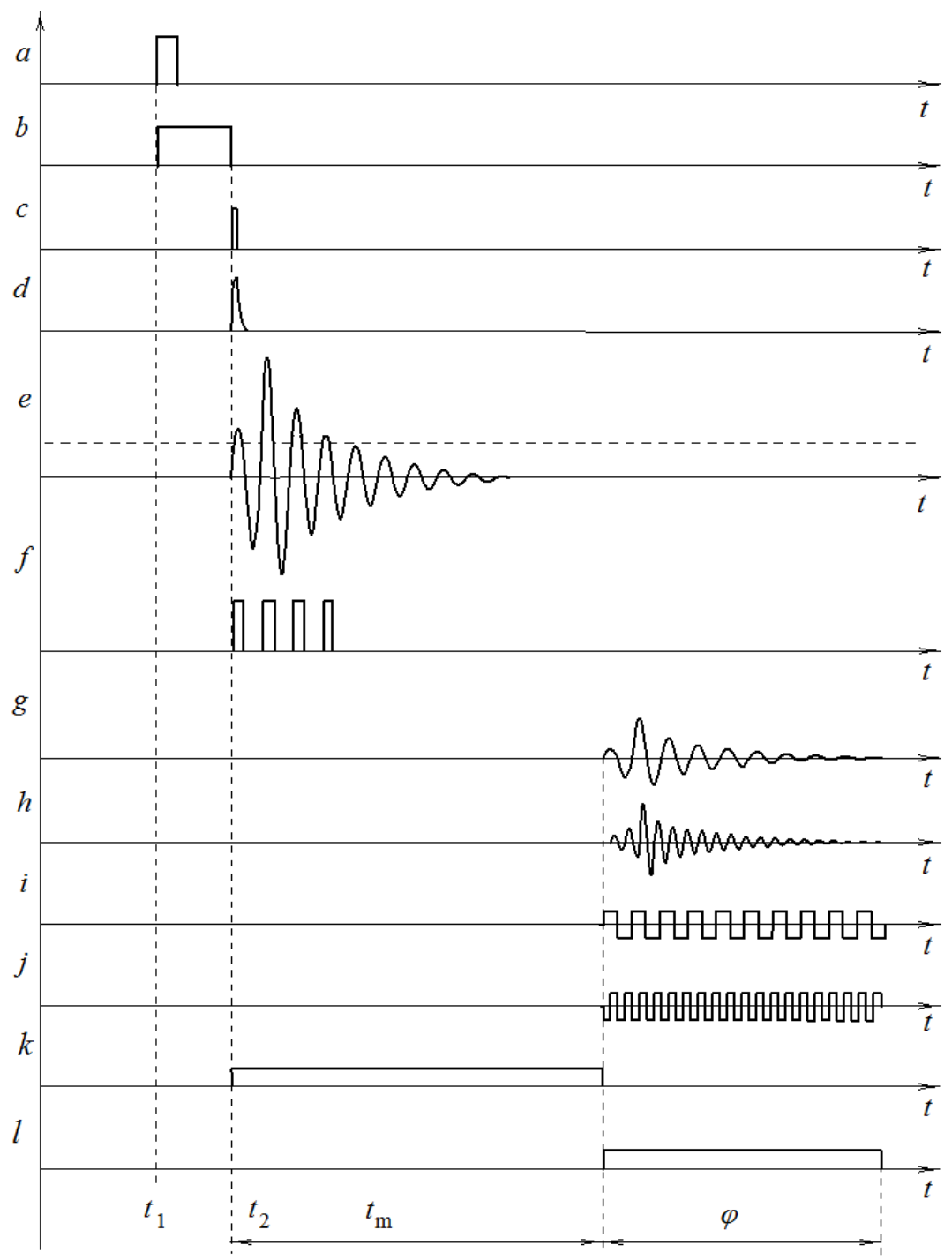

Fig. 2. Timing diagrams of the device

As a result, a high-voltage voltage pulse is generated at the output of the initiation system 24 , which acts on the input of the ignition device 23, after which, due to the acceleration of the flame front within the detonation generator 21, a shock wave is formed at its output.

When a shock wave is applied to the earth's surface, multifrequency harmonic damped seismic waves are excited, the waveforms and frequency composition of which depends on many factors, such as the nature of the impulse, the absorbing properties of the medium, and also the structural features of the interface in the wave path, and so on. In modern seismic exploration, powerful explosive sources are used that have pulse duration of several milliseconds, while the frequency band of the spectrum of the excited seismic waves is 1-200 Hz.

When detonation generators are used as an excitation source, the frequency band of the excited seismic waves expands several times, since the duration of the impact pulse in detonation generators can range from several tens of microseconds to several milliseconds, depending on the length of the detonation tube.

According to the above theory, the generated harmonic damped seismic wave on the surface of the earth has the form:

$$
P(t)=P_{1} e^{-\alpha_{1} t} \sin \left(\omega_{1} t\right)+P_{2} e^{-\alpha_{2} t} \sin \left(\omega_{2} t\right)+\ldots+P_{n} e^{-\alpha_{n} t} \sin \left(\omega_{n} t\right)
$$


where $P_{1}, P_{2}, \ldots, P_{n}$ - the maximum amplitude of harmonic components at frequencies $\omega_{1}, \omega_{2}, \ldots, \omega_{n}$, respectively; $\omega_{1}=2 \pi f_{1}, \omega_{2}=2 \pi f_{2}, \ldots, \omega_{n}=2 \pi f_{n}-$ circular frequency; $\alpha_{1}, \alpha_{2}, \ldots, \alpha_{n}-$ the attenuation coefficients of the medium corresponding to the frequencies $\omega_{1}, \omega_{2}, \ldots, \omega_{n}$, respectively; $t$ - time.

A timing diagram explaining the operation principle of the proposed device is shown in Fig. 2, where

a) signal of the generated sequence of short rectangular pulses in the synchronizer - 1 ;

b) rectangular pulse in the single oscillator -26 ;

c) rectangular pulse in the generator -25 ;

d) short pulses in the generator - 21;

e) signal in the initiation system - 24;

f) output pulse in the single oscillator - 26;

g) signal in the detonation generator -21 ;

h) signal in the radiation receiver -5 ;

i) signal in the threshold device -6 ;

g) signal in the frequency doubler - 10;

k) signal in the phase detector - 11;

1) signal in the time interval meter -14 .

From the theory of defect detection, it is known [5] that the basic information about the nature of the object is contained in the phase shift of the echo signals.

In accordance with this, a wave formed on the surface of the earth, spreading through the depth of the earth's crust, passes the distance $h_{c}$ and reaches the surface of the desired object reflected from it. The phases undergoing changes in each harmonic component pass once again the distance $h_{c}$ and reach the surface of the earth where the radiation receivers are installed. In this case, the wave acting on the radiation receiver has the form

$$
\begin{gathered}
P_{e}(t)=P_{e 1} e^{-\alpha_{1} t} \sin \left[\omega_{1}\left(t-\tau_{z}\right)+\phi_{1}\right]+P_{e 2} e^{-\alpha_{2} t} \sin \left[\omega_{2}\left(t-\tau_{z}\right)+\phi_{2}\right]+\ldots \\
\ldots+P_{e n} e^{-\alpha_{n} t} \sin \left[\omega_{n}\left(t-\tau_{z}\right)+\phi_{n}\right]
\end{gathered}
$$

where $\mathrm{P}_{\mathrm{e} 1}, \mathrm{P}_{\mathrm{e} 2}, \ldots, \mathrm{P}_{\mathrm{en}}$ the maximum amplitude of harmonic components of echo signals at frequencies $\omega_{1}, \omega_{2}, \ldots, \omega_{n}$, respectively; $\phi_{1}, \phi_{2}, \ldots, \phi_{n}$ - phase shift of echo signals at frequencies, respectively; $\tau_{\mathrm{z}}$ - delay time of echo signals corresponding at a distance of $2 \mathrm{~h}_{\mathrm{c}}$.

The electrical signal formed at the output of the radiation receiver has the form

$$
\begin{gathered}
\mathrm{U}_{\mathrm{e}}(\mathrm{t})=\mathrm{U}_{\mathrm{e} 1} \mathrm{e}^{-\alpha_{1} \mathrm{t}} \sin \left[\omega_{1}\left(\mathrm{t}-\tau_{\mathrm{z}}\right)+\phi_{1}\right]+\mathrm{U}_{\mathrm{e} 2} \mathrm{e}^{-\alpha_{2} \mathrm{t}} \sin \left[\omega_{2}\left(\mathrm{t}-\tau_{\mathrm{z}}\right)+\phi_{2}\right]+\ldots \\
\ldots+\mathrm{U}_{\mathrm{en}} \mathrm{e}^{-\alpha_{\mathrm{n}} \mathrm{t}} \sin \left[\omega_{\mathrm{n}}\left(\mathrm{t}-\tau_{\mathrm{z}}\right)+\phi_{\mathrm{n}}\right]
\end{gathered}
$$

where $\mathrm{U}_{\mathrm{e} 1}, \mathrm{U}_{\mathrm{e} 2}, \ldots, \mathrm{U}_{\mathrm{en}}$ - the maximum amplitude of the voltages of harmonic components of echo signals at frequencies $\omega_{1}, \omega_{2}, \ldots, \omega_{n}$, respectively.

According to the theory of acoustic control methods [7], to assess the nature of the object, it is sufficient to measure the phase shift between two neighboring harmonic components of the echo signals, which differ in frequency. The choice of a pair of harmonic components of echo signals is made by their more intense amplitudes, which in seismic exploration with the use of powerful explosive sources correspond to the frequency range of $20-80 \mathrm{~Hz}$ [8]. It is established that the use of detonation generators with a frequency of $20-400 \mathrm{~Hz}$ made it possible to determine and classify the desired object with a high accuracy at a depth of up to $100 \mathrm{~m}$.

It is known that in phasometry to measure phase shifts between components of harmonic signals they are reduced to one frequency by multiplying their original frequency [9, 10]. Then the phase shift between the selected harmonic components at the multiplicity of their frequency $\omega_{\mathrm{m}}=\mathrm{k} \omega_{0}, \omega_{(\mathrm{m}+1)}=(\mathrm{k}+1) \omega_{0}$, and when they are reduced to a single frequency, for example, $\omega=\mathrm{k}(\mathrm{k}+1) \omega_{0}$ by multiplying frequencies $\omega_{1}$ and $\omega_{(\mathrm{m}+1)}$, respectively on $(\mathrm{k}+1)$ and 
$\mathrm{k}$, allows to determine the phase difference between the harmonic components of the echo signals.

$$
\psi=(\mathrm{k}+1) \phi_{\mathrm{m}}-\mathrm{k} \phi_{(\mathrm{m}+1)}
$$

where $\mathrm{m}=1,2, \ldots-$ the number of the selected harmonic.

When the frequency of the selected harmonic components $\omega_{\mathrm{m}}$ and $\omega_{(\mathrm{m}+1)}$ is close, it can be set $\phi_{\mathrm{m}}=\phi_{(\mathrm{m}+1)}=\phi_{\mathrm{e}}$.

Therefore, the phase difference between harmonic components will be equal to the phase shift of the echo signal $\psi=\phi_{\mathrm{e}}$.

\section{Conclusions}

Echolocation device for geophysical exploration is intended for detection and primary classification of objects by their acoustic rigidity and can be used in hydroacoustics, fishing, namely for carrying out geophysical exploration at shallow depth.

In the device, the radiation source is made in the form of a detonation generator, a cooler is installed inside the mixer of the hydrocarbon air mixture of the detonation generator, and the combustion chamber of the detonation generator is provided with cooling radiators, which increase the reliability of geophysical exploration. It is established that the use of detonation generators with a frequency of $20-400 \mathrm{~Hz}$ made it possible to determine and classify the required objects with a high accuracy at a depth of up to $100 \mathrm{~m}$.

\section{References}

[1] Danko, D. A. (2016). Comparison of deterministic acoustic inversion methods for prediction of the prospective acoustically contrast objects from seismic data. The Russian Geophysics Journal, 1, 2-11.

[2] Belousov, A. V., Kostitsyn, V. I. (2015). Itogi mezhdunarodnoi nauchno-prakticheskoi konferentsii “Teoriia i praktika razvedochnoi i promyslovoi geofiziki”. Geofizicheskii vestnik, 6, 9-12.

[3] Karapetov, G. A. (2013). Spectro-holographic method of velocity analysis. The Russian Geophysics Journal, 5, 68-74.

[4] Davydov, A. B., Skovorodnikov, I. G., Davydov, V. A. (1995). Sposob gamma-oprobovaniia gornyh porod i rud na soderzhanie radioaktivnyh elementov. Informatsionno-tematicheskii sbornik RF. Ekaterinburg: IG RIA, 18-29.

[5] Davydov, V. A. (2010). Use of shallow seismic investigation for studying of undermined areas. News of the Higher Institutions. Mining Journal, 4, 111-116.

[6] Park, C. B., Miller, R. D., Xia, J., Ivanov, J. (2007). Multichannel analysis of surface waves (MASW) - active and passive methods. The Leading Edge, 26 (1), 60-64. doi: 10.1190/1.2431832

[7] Pirttijärvi, M. T., Lerssi, J. (2006). Laterally Constrained 1D Inversion of Airborne Electromagnetic Data. Near Surface 2006 - 12th EAGE European Meeting of Environmental and Engineering Geophysics. doi: 10.3997/2214-4609.201402626

[8] Thitimakorn, T., Anderson, N. L., Stephenson, R., Liu, W. (2005). 2-D Shear-Wave Velocity Profile Along Test Segment of Interstate I-70, St. Louis, Missouri. Site Characterization and Modeling. doi: 10.1061/40785(164)14

[9] Garcia, X., Jones, A. G. (2002). Atmospheric sources for audio-magnetotelluric (AMT) sounding. GEOPHYSICS, 67 (2), 448-458. doi: 10.1190/1.1468604

[10] Hayashi, K., Suzuki, H. (2004). CMP cross-correlation analysis of multi-channel surface-wave data. Exploration Geophysics, 35 (1), 7. doi: 10.1071/eg04007 